TI 2006-048/2

Tinbergen Institute Discussion Paper

\title{
Are Economic Agents Successful Optimizers? \\ An Analysis through Service Strategy in Tennis
}

Franc J.G.M. Klaassen'

Jan R. Magnus²

1 University of Amsterdam, and Tinbergen Institute;

2 Tilburg University. 


\section{Tinbergen Institute}

The Tinbergen Institute is the institute for economic research of the Erasmus Universiteit Rotterdam, Universiteit van Amsterdam, and Vrije Universiteit Amsterdam.

Tinbergen Institute Amsterdam

Roetersstraat 31

1018 WB Amsterdam

The Netherlands

Tel.: $\quad+31(0) 205513500$

Fax: $\quad+31(0) 205513555$

Tinbergen Institute Rotterdam

Burg. Oudlaan 50

3062 PA Rotterdam

The Netherlands

Tel.: $\quad+31(0) 104088900$

Fax: $\quad+31(0) 104089031$

Please send questions and/or remarks of nonscientific nature to driessen@tinbergen.nl.

Most TI discussion papers can be downloaded at http://www.tinbergen.nl. 


\title{
Are economic agents successful optimizers? An analysis through service strategy in tennis
}

\author{
Franc J.G.M. Klaassen \\ Tinbergen Institute and University of Amsterdam, The Netherlands \\ Jan R. Magnus \\ Department of Econometrics 8 OR, Tilburg University, The Netherlands
}

May 19, 2006

JEL Classification: C14, C15, D01, D21

Key words: Inefficiency, Frontier, Optimal strategy, Tennis.

\begin{abstract}
We consider the question whether top tennis players in a top tournament (Wimbledon) employ an optimal (efficient) service strategy. We show that top players do not, in general, follow an optimal strategy, and we provide a lower bound of the inefficiency. The inefficiency regarding winning a point on service is on average at least $1.1 \%$ for men and $2.0 \%$ for women, leading to a possible increase of income for the efficient player of $18.7 \%$ for men and $32.8 \%$ for women. We use these findings to shed some light on the question whether economic agents are successful optimizers.
\end{abstract}

Corresponding author: Jan R. Magnus, Department of Econometrics \& Operations Research, Tilburg University, P.O. Box 90153, 5000 LE Tilburg, The Netherlands. Phone: +31-13-466-3092, fax: +31-13-466-3066, e-mail: magnus@uvt.nl. 


\section{Introduction}

Most economists believe that agents maximize something and that they are successful in doing so. Stigler (1976) provides a typical and outspoken example of such a view in his critique of Leibenstein's (1966) notion of X-efficiency. Any inefficiency claimed by Leibenstein or others is - according to Stigler - nothing but a failure to measure all relevant inputs, or to correctly specify what is being maximized. For example, John Capozzi's well-known business maxim:

Only make a great deal if you have no intention of ever doing business with that person again... otherwise make a good deal,

would not - if followed - indicate inefficiency. It might indicate that the firm does not maximize short-term profit, but the firm would still maximize long-term profit or market power or "utility." Førsund, Lovell and Schmidt (1980, p. 21) point out that such a view is essentially an act of faith, as it can be neither proved nor disproved.

Perhaps, however, we can prove or disprove the hypothesis that agents are successful maximizers. For this we would need a situation where (a) it is unambiguous what it is that the agent wishes to maximize, and (b) clean and complete data are available. Under these conditions any apparent suboptimality must be true suboptimality. Such a situation can only be found in a very structured environment. One possibility for creating such an environment is through a laboratory experiment. This has the advantage of maximum control, but it also has disadvantages. Instead we have chosen a "field experiment" as our environment: the service strategy of tennis players at Wimbledon. This is a real-life setting where high prizes can be won and where the participants are highly trained and very experienced. Thus we study not top firms but top tennis players.

Tennis is a highly competitive sport and the top players (our agents) want to win matches on the professional tour, especially at the "grand slam" tournaments of which Wimbledon is arguably the most important. It seems reasonable to assume that these agents wish to maximize the probability of winning a match. In addition, our data are clean. The tennis environment is therefore ideal for our purpose. Based on a simple model we can calculate the players' optimal strategy and we can compare this with their actual strategy. The discrepancy (ratio) between the two defines their efficiency. We intend to show that tennis players are not entirely successful in maximizing their objective function and we shall provide a lower bound of the inefficiency.

Given the difficulty of establishing efficiency using economic data, the principal question in efficiency analysis for firms (or other institutions or in- 
dividuals) is to establish relative efficiency: how well does one firm perform relative to another firm, in particular relative to a top firm. There exist various well-established techniques to measure relative efficiency, in particular data envelopment analysis (DEA), developed by Charnes, Cooper and Rhodes (1978), and widely employed in management science. In contrast, we shall be interested in absolute efficiency by asking: how well do agents perform compared to the optimum achievable. Thus we shall not assume that top agents lie on the efficiency frontier. Instead we want to measure how far they are removed from the frontier.

Several methods have been proposed to allow for inefficiencies of firms, in particular stochastic frontier analysis (SFA), see the surveys by Førsund, Lovell and Schmidt (1980) and Schmidt (1985/86), the monograph by Kumbhakar and Lovell (2000), and a Bayesian perspective by Koop, Osiewalsky and Steel (1997). The main emphasis of these studies is the measurement of efficiency (productivity) of an average firm, taking into account measurement error and inefficiency at the frontier. The interest is not primarily on estimating the inefficiency of the top firm and indeed estimates of such inefficiencies are hard to find.

Our interest, however, is entirely focussed on the top agents, not on average agents. We want to know whether top tennis players are efficient and, if not, how much room for improving their efficiency exists.

Sports statistics (and sports economics) has developed from an anecdotal field where one collects statistics (so many double faults, so many aces), to an almost-respectable discipline. An important reason for this development is that sport statistics can help answer behavioral questions. Moreover, sports data are readily available and they are measured much more precisely than most economic data. This has led to studies on racial discrimination (Gwartney and Haworth, 1974; Kahn and Sherer, 1988; Nardinelli and Simon, 1990; Stone and Warren, 1999; Szymanski, 2000; Kanazawa and Funk, 2001; and Goff, McCormick and Tollison, 2002), efficiency of the betting market (Zuber, Gandar and Bowers, 1985; Sauer, Brajer, Ferris and Marr, 1988; Golec and Tamarkin, 1991; Dixon and Coles, 1997; and Gray and Gray, 1997), comparison of betting markets and financial markets (Levitt, 2004), the effect of labor strikes on consumer demand (Schmidt and Berri, 2004), preferences under risk (Julien and Salanié, 2000), mixed strategy equilibria (Walker and Wooders, 2001; and Chiappori, Levitt and Groseclose, 2002), incentive effects (Ehrenberg and Bognanno, 1990), rationality (Gandar, Zuber, O'Brian and Russo, 1988), optimal labor contracts (Lazear and Rosen, 1981), control of externalities (Carlton, Frankel and Landes, 2004), favoritism (Garicano, Palacios-Huerta and Prendergast, 2005), and so on. 
One possible problem with the application of sports data is that they typically apply to top athletes and that top athletes behave rather differently than average athletes. Hence to use sports data to say something about top firms (as we shall do) may be reasonable, whereas the application to average firms may be less reasonable. Nevertheless, we shall show that inefficiencies are larger for the relatively weak top players.

To study efficiency of human behavior, tennis data are particularly useful. This is because tennis has an unusual and archaic rule which does not exist in other comparable sports (table tennis, badminton, volleyball), namely that the server has two chances to bring the ball into play (first service, second service) rather than one. Even with one service the question needs to be answered how difficult to make this service: too easy and the server loses the point in the subsequent rally, too difficult and the service will be a fault much of the time. Choosing the right balance is obviously important. In the current situation the server has two services, and this has two consequences. First, to determine the optimal strategy of choosing the strengths of the two services is more difficult than in the one-service case. Second, the existence of two services doubles the amount of information we possess about a player's strategy against a specific opponent.

The organization of this paper is as follows. In Section 2 we present the theoretical model, based on the relationship between the probability that a service is in $(x)$ and the conditional probability that the server wins the point if the service is in $(y(x))$. We prove the existence and uniqueness of an optimal strategy and introduce the concept of efficiency of the service. In Section 3 we propose a functional form for $y(x)$. Since tennis allows two services, we have information on the (current, not necessarily optimal) strategy $\left(x_{1}, x_{2}\right)$ and the corresponding "yield" $\left(y_{1}, y_{2}\right)$. We do not, however, observe these probabilities directly, only the associated relative frequencies. In Section 4 we estimate the key probabilities using a generalized method of moments (GMM) approach. Next, in Section 5 we discuss the identification and estimation of the curvature of the $y$-curve. Then, in Section 6, we estimate and discuss the (in)efficiencies of top tennis players. In our conclusion we try to relate these results to the inefficiency of economic agents.

\section{Theoretical model}

Consider a tennis match between two players $i$ and $j$. Both $i$ and $j$ are maximizing the probability of winning the match. If points are independent, then each server chooses that service strategy which will maximize the probability of winning a point. We will develop a model that answers the question how 
difficult a player should make his or her service in order to maximize the probability of winning a point on service.

\subsection{Existence and uniqueness of an optimal strategy}

Given the strengths of both players, let $x_{1}$ denote the probability that the first service is in, and let $x_{2}$ denote the probability that the second service is in. Also, let $y(x)$ denotes the conditional probability that player $i$ wins the point if the service has probability $x$ of being in. So, $y_{1}:=y\left(x_{1}\right)$ denotes the conditional probability that player $i$ wins the point on his or her first service if the first service is in, and similarly for the second service $y_{2}:=y\left(x_{2}\right)$. We realize that both $x$ and $y$ will be influenced by other factors (speed, direction, spin). For the moment we abstract from these unobservable factors, but we shall discuss them in Section 2.3.

Given $x$ and $y(x)$ we define $w(x):=x \cdot y(x)$, which transforms the conditional probability $y$ into an unconditional probability $w$. The probability that player $i$ wins the point is then given by

$$
p\left(x_{1}, x_{2}\right)=w\left(x_{1}\right)+\left(1-x_{1}\right) w\left(x_{2}\right) .
$$

If the functional form of $y(x)$ is known, we can calculate the optimal service strategy $\left(x_{1}^{*}, x_{2}^{*}\right)$ by maximizing $p\left(x_{1}, x_{2}\right)$. Under suitable regularity conditions and in the absence of a boundary solution, the optimal strategy satisfies the first-order conditions

$$
w^{\prime}\left(x_{1}^{*}\right)=w\left(x_{2}^{*}\right), \quad w^{\prime}\left(x_{2}^{*}\right)=0 .
$$

In what follows we shall, however, allow for the possibility of a boundary solution $x_{2}^{*}=1$.

It seems reasonable to assume that the easier a player makes his or her service, the more likely it is that the service is in ( $x$ increases), but the less likely it is that the point is won if the service is in ( $y$ decreases). Hence, we impose the condition that $y$ is a decreasing function of $x$.

Condition R1 (monotonicity): The real-valued function $y$ is continuous and monotonically decreasing on [0,1], and satisfies $0 \leq y(x) \leq 1$ for all $x \in[0,1]$.

In order to achieve existence and uniqueness of the optimal strategy we need more than monotonicity. We shall also impose (local) concavity. 
Condition R2 (concavity): The real-valued function $y$ is twice differentiable on $(0,1)$ with $y^{\prime}(1):=\lim _{x \uparrow 1} y^{\prime}(x)<0$, and $w(x)$ satisfies $w^{\prime \prime}(x)<0$ for all $x \in(0,1)$.

Condition R2 implies that $w$ is strictly concave on the interval $(0,1)$ and reaches a unique maximum for some $x \in(0,1]$. This reflects the fact that if a player's service is too easy he/she is unlikely to win the point, but if the service is too difficult he/she is also unlikely to win the point - in fact, $w(0)=0$. There should be an optimal service, neither too difficult nor too easy, which maximizes the player's probability of winning the point on that service. Given these regularity conditions we can now prove Theorem 1.

Theorem 1 (existence and uniqueness): Assume that regularity conditions $\mathrm{R} 1$ and $\mathrm{R} 2$ hold. Then there exists a unique optimal service strategy $\left(x_{1}^{*}, x_{2}^{*}\right)$ which maximizes $p\left(x_{1}, x_{2}\right)$.

Proof. Both steps of the proof use the fact that $w^{\prime}$ is monotonically decreasing. For the first step (regarding $x_{2}^{*}$ ) we note that $w^{\prime}(0):=\lim _{x \downarrow 0} w^{\prime}(x)=$ $y(0)>0$. If $w^{\prime}(1):=\lim _{x \uparrow 1} w^{\prime}(x)<0$, then there exists a unique $x_{2}^{*} \in(0,1)$ such that $w^{\prime}\left(x_{2}^{*}\right)=0$. If $w^{\prime}(1) \geq 0$, then $w(x)$ is monotonically increasing for all $x$ and hence reaches its maximum at $x_{2}^{*}=1$.

For the second step (regarding $x_{1}^{*}$ ) we show first that $w\left(x_{2}^{*}\right)>w^{\prime}\left(x_{2}^{*}\right)$. If $w^{\prime}(1)<0$, then $w^{\prime}\left(x_{2}^{*}\right)=0$ and $w\left(x_{2}^{*}\right)>0$, and hence $w\left(x_{2}^{*}\right)>w^{\prime}\left(x_{2}^{*}\right)$. If $w^{\prime}(1) \geq 0$, then $x_{2}^{*}=1$ and

$$
w\left(x_{2}^{*}\right)=w(1)=y(1)>y(1)+y^{\prime}(1)=w^{\prime}(1)=w^{\prime}\left(x_{2}^{*}\right),
$$

because $y^{\prime}(1)<0$. In addition,

$$
w\left(x_{2}^{*}\right)=x_{2}^{*} y\left(x_{2}^{*}\right) \leq y\left(x_{2}^{*}\right)<y(0)=w^{\prime}(0) .
$$

Thus we find that

$$
w^{\prime}\left(x_{2}^{*}\right)<w\left(x_{2}^{*}\right)<w^{\prime}(0)
$$

and hence there exists a unique $x_{1}^{*}$ with $0<x_{1}^{*}<x_{2}^{*}$ such that $w^{\prime}\left(x_{1}^{*}\right)=$ $w\left(x_{2}^{*}\right)$. $\|$

We remark that condition $\mathrm{R} 2$ (global concavity) imposes a strong restriction on the $y$-curve. In fact, condition $\mathrm{R} 2$ can be much weakened. First, we note from the proof that all that is required is that $\mathrm{R} 2$ holds locally for all $x \in\left(x_{0}, 1\right)$, where $0 \leq x_{0}<x_{1}^{*}$, and this is in fact what we shall use in our estimation. (The choice $x_{0}=0.4$ appears to be satisfactory.) Second, 
we may wish to choose the $y$-curve such that it gives us maximum flexibility over the slopes at $x_{1}^{*}$ and $x_{2}^{*}$. This is possible because all we need is that there exists a pair $\left(x_{1}^{*}, x_{2}^{*}\right)$ with $0<x_{1}^{*}<x_{2}^{*} \leq 1$, such that $w(x)$ has a unique maximum at $x_{2}^{*}$ and $w(x)+(1-x) w\left(x_{2}^{*}\right)$ has a unique maximum at $x_{1}^{*}$. The $y$-curve may thus have kinks. We shall use this generalization later when we discuss the robustness of the specification.

\subsection{Implications}

Theorem 1 has certain implications for the optimal service strategy $\left(x_{1}^{*}, x_{2}^{*}\right)$; see also Gale (1971).

Theorem 2 (implications): If conditions R1 and R2 are satisfied, then the following relations must hold for the optimal strategy $\left(x_{1}^{*}, x_{2}^{*}\right)$ :

(a) $x_{1}^{*}<x_{2}^{*}$

(b) $y\left(x_{1}^{*}\right)>y\left(x_{2}^{*}\right)$

(c) $w\left(x_{1}^{*}\right)<w\left(x_{2}^{*}\right)$, and

(d) $w\left(x_{2}^{*}\right)-w\left(x_{1}^{*}\right)<\left(x_{2}^{*}-x_{1}^{*}\right) w\left(x_{2}^{*}\right)$.

Proof. Statement (a) is implied in the proof of Theorem 1. Statement (b) then follows since $y$ is a decreasing function. Statement (c) follows from the fact that if $\left(x_{1}^{*}, x_{2}^{*}\right)$ is the optimal service strategy, then this strategy should lead to a higher probability of winning the point than the strategy $\left(x_{1}^{*}, x_{1}^{*}\right)$ (always using the first service), that is,

$$
w\left(x_{1}^{*}\right)+\left(1-x_{1}^{*}\right) w\left(x_{2}^{*}\right)>w\left(x_{1}^{*}\right)+\left(1-x_{1}^{*}\right) w\left(x_{1}^{*}\right),
$$

and this simplifies to (c). Similarly, the optimal strategy $\left(x_{1}^{*}, x_{2}^{*}\right)$ should lead to a higher probability of winning the point than the strategy $\left(x_{2}^{*}, x_{2}^{*}\right)$ (always using the second service). This implies

$$
w\left(x_{1}^{*}\right)+\left(1-x_{1}^{*}\right) w\left(x_{2}^{*}\right)>w\left(x_{2}^{*}\right)+\left(1-x_{2}^{*}\right) w\left(x_{2}^{*}\right)
$$

which simplifies to $(\mathrm{d})$. ॥

Relations (a) and (b) say that the first service should be more difficult than the second in two senses: (a) it is less often in, and (b) if it is in it is more likely to win the point. Relation (c) is less obvious. It says that the probability of winning a point at the first (difficult) service should be smaller than the probability of winning a point on the second (easier) service. 
As a preliminary exercise, we calculate the observed frequencies for each player in each match using the data described in Section 4.1.

If a player serves optimally, he or she should satisfy the four implications stated in Theorem 2. In fact, these implications are often not satisfied.

Table 1-Four Consistency Conditions

\begin{tabular}{cccccc}
\hline \hline & (a) & (b) & (c) & (d) & Total \\
\hline Men & 1.00 & 0.91 & 0.78 & 0.80 & 0.59 \\
Women & 0.98 & 0.72 & 0.77 & 0.64 & 0.42 \\
\hline
\end{tabular}

The condition $x_{1}<x_{2}$ appears to be almost always satisfied, which means that almost all players take more risk on their first service than on their second service (as they should). However, this additional risk does not necessarily translate into higher productivity: the condition $y\left(x_{1}\right)>y\left(x_{2}\right)$ is only satisfied for $91 \%$ of the men and $72 \%$ of the women. Condition (c) requires that $\left(x_{1}, x_{2}\right)$ is a better service strategy than $\left(x_{1}, x_{1}\right)$, but this is only true for $77-78 \%$ of the players. Condition (d) requires that $\left(x_{1}, x_{2}\right)$ is a better service strategy than $\left(x_{2}, x_{2}\right)$, but this is only true for $80 \%$ of the men and $64 \%$ of the women. For only $59 \%$ of the men and $42 \%$ of the women are all four consistency requirements satisfied.

It therefore seems that for many players the probability of winning a point can be increased by making small changes to their service strategy. Indeed, since the four consistency conditions are necessary but not sufficient for an optimal strategy (and hence even if all four conditions are satisfied a player may not follow the optimal strategy), the actual deviations from the optimum will be even larger. This conclusion, however, is too hasty and simplistic. For one, there will be measurement error: we are interested in probabilities but we observe relative frequencies, and this might lead to smaller deviations from the optimum. A more careful statistical analysis is required.

\subsection{Other influences}

It would be naive to believe that the $y$-curve only depends on $x$. In fact, both $x$ and $y$ will depend on a $p \times 1$ vector $\boldsymbol{\omega}$ representing speed, direction, spin, et cetera. We define an auxiliary vector $\boldsymbol{v}=\left(v_{1}, \ldots, v_{p-1}\right)^{\prime}$ such that $(x, \boldsymbol{v})$ and $\boldsymbol{\omega}$ are in one-to-one correspondence, and we write $y=y(x, \boldsymbol{v})$. Suppose we have only one service. Then we should maximize $w(x, \boldsymbol{v}):=x \cdot y(x, \boldsymbol{v})$ with respect to $x$ and $\boldsymbol{v}$. The first-order conditions are

$$
y(x, \boldsymbol{v})+x \frac{\partial y(x, \boldsymbol{v})}{\partial x}=0, \quad \varphi_{h}(x, \boldsymbol{v}):=\frac{\partial y(x, \boldsymbol{v})}{\partial v_{h}}=0 \quad(h=1, \ldots, p-1) .
$$


For given $x$, assume that the equations $\varphi_{h}(x, \boldsymbol{v})=0$ have a unique solution $\boldsymbol{v}=\boldsymbol{\zeta}(x)$, so that each $\varphi_{h}(x, \boldsymbol{\zeta}(x))=0$ for all $x$. At the optimum $\left(x^{*}, \boldsymbol{v}^{*}\right)$ we have $\varphi_{h}\left(x^{*}, \boldsymbol{v}^{*}\right)=0$, and hence

$$
\begin{aligned}
\left.\frac{\partial y(x, \boldsymbol{\zeta}(x))}{\partial x}\right|_{x=x^{*}} & =\left.\frac{\partial y\left(x, \boldsymbol{\zeta}\left(x^{*}\right)\right)}{\partial x}\right|_{x=x^{*}}+\left.\sum_{h=1}^{p-1} \varphi_{h}\left(x^{*}, \boldsymbol{v}^{*}\right) \frac{\partial \zeta_{h}(x)}{\partial x}\right|_{x=x^{*}} \\
& =\left.\frac{\partial y\left(x, \boldsymbol{\zeta}\left(x^{*}\right)\right)}{\partial x}\right|_{x=x^{*}} .
\end{aligned}
$$

The same reasoning applies when there are two services. We conclude that we may think of the $y$-curve as $y(x, \boldsymbol{\zeta}(x))$, where $\boldsymbol{v}=\boldsymbol{\zeta}(x)$ is chosen optimally for given $x$. Obviously some assumption is required on $\boldsymbol{v}$, since no data are available on either $\boldsymbol{v}$ or $\boldsymbol{\omega}$. The particular assumption of choosing $\boldsymbol{v}$ optimally implies a "conservative" estimate of the efficiency. The same applies to the identification and estimation of $\lambda$ (the curvature parameter of the $y$-curve), as we shall see in Section 5: no data and a conservative approach. We shall come back to the effects of the conservative approach when we discuss our efficiency results in Section 6.

\subsection{Efficiency}

In a given match between two players the probability that the server wins a point is given by (1):

$$
p=x_{1} y_{1}+\left(1-x_{1}\right) x_{2} y_{2} .
$$

Whether or not $\left(x_{1}, y_{1}\right)$ and $\left(x_{2}, y_{2}\right)$ are the optimal probabilities, the $y$ curve will pass through these two points. If the $y$-curve were linear then the two points would determine the curve, but we shall see that linearity is too restrictive. Suppose therefore that the $y$-curve depends on one (or more) curvature parameter $\lambda$. Given the $y$-curve, we obtain the optimal strategy $\left(x_{1}^{*}, x_{2}^{*}\right)$ and the corresponding function values $y_{1}^{*}=y\left(x_{1}^{*}\right)$ and $y_{2}^{*}=y\left(x_{2}^{*}\right)$, all of which depend on $\lambda$. The maximum probability of winning a point is thus

$$
p^{*}=x_{1}^{*} y_{1}^{*}+\left(1-x_{1}^{*}\right) x_{2}^{*} y_{2}^{*},
$$

and we define the efficiency of the server in this match as

$$
\text { eff }:=p / p^{*}
$$

which is a number between zero and one. The closer eff is to one, the higher is the efficiency. Note that the efficiency differs per player.

Of course, we do not know the probabilities $\left(x_{1}, y_{1}\right)$ and $\left(x_{2}, y_{2}\right)$ and the curvature parameter $\lambda$. Their estimation is taken up in Sections 4 and 5 . But first we need to discuss the specification of the $y$-curve. 


\section{$3 \quad$ Functional form for $y(x)$}

The simplest specification for $y$ is a linear function. This, however, does not work well in practice. For example, it forces $x_{1}^{*} \leq 1 / 2$, which is not realistic since the observed frequencies for $x_{1}$ are $59.5 \%$ for men and $61.6 \%$ for women. Some curvature is required. We propose the following simple nonlinear function:

$$
y(x)=\frac{\alpha-x^{\lambda}}{\tau}, \quad \lambda>0 .
$$

The two regularity conditions $\mathrm{R} 1$ and $\mathrm{R} 2$ are satisfied if and only if

$$
1 \leq \alpha \leq \tau+x_{0}^{\lambda}
$$

where $0 \leq x_{0}<x_{1}^{*}$. The proposed $y$-curve allows $y$ to be either concave or convex (or linear) on the $(0,1)$ interval, depending on whether $\lambda>1$ or $0<\lambda<1$ (or $\lambda=1$ ). Given (6) we obtain $w(x)=x\left(\alpha-x^{\lambda}\right) / \tau$. If $\alpha<\lambda+1$, then $w^{\prime}(1)<0$ and $x_{2}^{*}$ is obtained from the first-order condition

$$
w^{\prime}\left(x_{2}^{*}\right)=\frac{\alpha-(\lambda+1) x_{2}^{* \lambda}}{\tau}=0,
$$

so that $x_{2}^{* \lambda}=\alpha /(\lambda+1)$. If $\alpha \geq \lambda+1$, then $w^{\prime}(1) \geq 0$ and $x_{2}^{*}=1$. Hence,

$$
x_{2}^{* \lambda}=\min \left(\frac{\alpha}{\lambda+1}, 1\right) .
$$

Given $x_{2}^{*}$, we find $x_{1}^{*}$ from the equation $w^{\prime}\left(x_{1}^{*}\right)=w\left(x_{2}^{*}\right)$, that is

$$
\alpha-(\lambda+1) x_{1}^{* \lambda}=x_{2}^{*}\left(\alpha-x_{2}^{* \lambda}\right)
$$

from which we solve

$$
x_{1}^{* \lambda}=x_{2}^{* \lambda}\left(1-\frac{\lambda}{\lambda+1} x_{2}^{*}\right) .
$$

From $\left(x_{1}^{*}, x_{2}^{*}\right)$ we immediately obtain $y_{1}^{*}=y\left(x_{1}^{*}\right)$ and $y_{2}^{*}=y\left(x_{2}^{*}\right)$.

The geometry of the solution is illustrated in Figure 1, where we have chosen $\alpha=2.4, \tau=3.0$, and $\lambda=3.0$. On the horizontal axis we find the probability $x$ that the service is in; on the vertical axis we find the conditional probability $y(x)$ that server wins the point if the service is in, the unconditional probability $w(x)=x \cdot y(x)$ that server wins the point on this service, and the derivative $w^{\prime}(x)$. The optimal service strategy is found from Equations (8) and (9) which yields $x_{1}^{*}=60.4 \%$ and $x_{2}^{*}=84.3 \%$. If the optimal strategy is employed, the probability of winning a point on service 


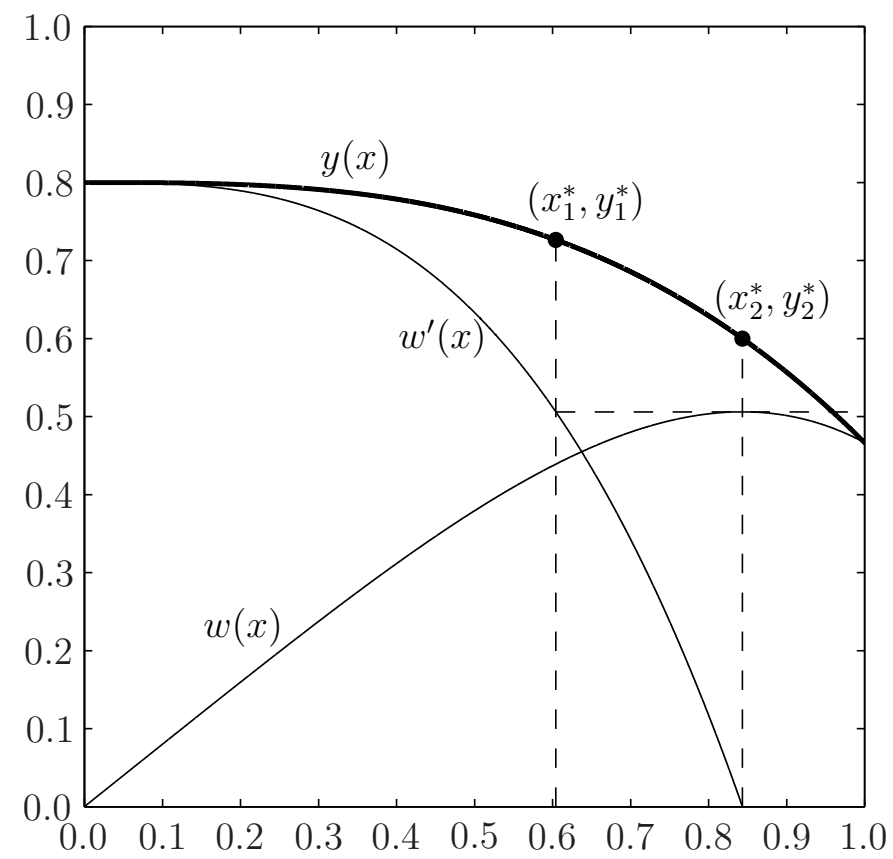

Figure 1. Optimal service strategy.

is $p^{*}=63.9 \%$, the probability of a double fault is $6.2 \%$, and the probability of winning a service game is $81.1 \%$.

Suppose we have observed $x_{1}, x_{2}, y_{1}$, and $y_{2}$ for the server in a given match. This gives us two points $\left(x_{1}, y_{1}\right)$ and $\left(x_{2}, y_{2}\right)$ on the curve. For each $\lambda$ we can solve $\tau$ and $\alpha$ from the two equations

$$
\frac{\alpha-x_{1}^{\lambda}}{\tau}=y_{1}, \quad \frac{\alpha-x_{2}^{\lambda}}{\tau}=y_{2} .
$$

The solution reads

$$
\tau=\frac{x_{2}^{\lambda}-x_{1}^{\lambda}}{y_{1}-y_{2}}, \quad \alpha=\frac{y_{1} x_{2}^{\lambda}-y_{2} x_{1}^{\lambda}}{y_{1}-y_{2}} .
$$

Inserting (11) into (6) then gives

$$
y(x)=\frac{y_{1} x_{2}^{\lambda}-y_{2} x_{1}^{\lambda}}{x_{2}^{\lambda}-x_{1}^{\lambda}}-\frac{y_{1}-y_{2}}{x_{2}^{\lambda}-x_{1}^{\lambda}} \cdot x^{\lambda} .
$$

Hence, in order to compute the $y$-curve and the optimal service strategy, we need to estimate $\lambda$ and the probabilities $\left(x_{1}, y_{1}\right)$ and $\left(x_{2}, y_{2}\right)$; that is, the probabilities actually employed by the player. The method of estimating 
$\left(x_{1}, y_{1}\right)$ and $\left(x_{2}, y_{2}\right)$ is outlined in Section 4. The parameter $\lambda$ is essentially unidentified from the data, and we shall discuss this problem (and a way round it) in Section 5.

\section{Estimation of the key probabilities}

In Section 2 we highlighted the importance of the two probabilities $x$ (probability that service is in) and $y$ (conditional probability that server wins the point if service is in). For player $i$ against player $j$ the key probabilities are given by

$$
\boldsymbol{z}_{i j}:=\left(x_{1, i j}, x_{2, i j}, y_{1, i j}, y_{2, i j}\right)^{\prime},
$$

where $x_{1, i j}$ denotes the probability that $i$ (playing against $j$ ) serves his or her first service in, and similarly for the other three probabilities. We cannot observe these four key probabilities, but we can observe the associated relative frequencies, denoted by $\boldsymbol{f}_{i j}$, and the associated number of observations underlying each of the relative frequencies, denoted by $\boldsymbol{t}_{i j}$. If the $\boldsymbol{t}_{i j}$-dimension were sufficiently large, then the probabilities in $\boldsymbol{z}_{i j}$ would be well approximated by the relative frequencies in $\boldsymbol{f}_{i j}$, and there would be no need to model $\boldsymbol{z}_{i j}$ further. In that case we could jump directly to Section 5 and treat $\left(\boldsymbol{z}_{i j}, \boldsymbol{z}_{j i}\right)$ as given. However, the $\boldsymbol{t}_{i j}$-dimension is not large, especially for the second service where (in our data set) it gets as low as 14 (men) and 5 (women). Hence important efficiency gains can be achieved by modeling $\boldsymbol{z}_{i j}$.

\subsection{The data}

Our data consist of singles matches played at Wimbledon during 1992-1995: 508 matches for the men and 508 matches for the women. For each of these matches we know the two players, their rankings at the beginning of the tournament, and the match result. For almost half of the matches (258 matches in the men's singles and 223 matches in the women's singles) we know the complete sequence of points. The data are described in detail in Magnus and Klaassen (1999a).

The reason that we do not have detailed data on all matches played during the four years is that only matches played on one of the five "show courts" (Centre Court and Courts 1, 2, 13, and 14) have been recorded. Typically, matches involving the most important players are scheduled on the show courts, and this causes an under-representation in the data set of matches involving weaker players. All results in this section have been corrected for this selection problem by weighting the matches by the inverses 
of the sampling percentages. The weighting procedure is discussed in detail in Magnus and Klaassen (1999b).

In the current section we need sixteen summary statistics per match (although we need more match information in some of the sensitivity analysis). For player $i$ serving against player $j$ we use the relative frequencies $\boldsymbol{f}_{i j}$ and the associated numbers of observations $\boldsymbol{t}_{i j}$; and the same for player $j$ serving against $i$. For example, suppose player $i$ serves 100 times against player $j$. Of the 100 first services, 60 are in and 40 are a fault; if the first service is in, player $i$ wins the point in 44 cases and loses it in 16 cases. Of the 40 second services, 35 are in and 5 are a fault (double fault); if the second service is in, player $i$ wins the point in 21 cases and loses it in 14 cases. This information allows us to compute $\boldsymbol{f}_{i j}=(0.600,0.875,0.733,0.600)^{\prime}$ and $\boldsymbol{t}_{i j}=(100,40,60,35)^{\prime}$.

We do not have access to more matches of which all relevant summary statistics are known. If we had, this would have decreased the estimation uncertainty from the GMM procedure described below. We could have increased the number of matches where we only require the rankings (currently 508 matches) by including Wimbledon singles matches from 1996 onwards. This would, however, have added nothing and it would also have raised the question of parameter instability over time.

\subsection{The measurement equation}

In what follows we assume that matches are independent (even when servers occur in several matches), and that points played in one match are independent and identically distributed (i.i.d.). The independence of matches seems a reasonable assumption, but the i.i.d. assumption of points within one match is a hot issue (not only in tennis), and was analyzed by Klaassen and Magnus (2001). They conclude that points in tennis are neither independent nor identically distributed, but that the deviation is small (though statistically significant) and that therefore the i.i.d. assumption will still be reasonable in many specific directions. In addition, in our case, we do not use the points themselves but summary statistics (averages) so that any possible harm caused by the wrong assumption is much reduced.

In measuring the (nonobservable) random probability $\boldsymbol{z}_{i j}$ with the (observable) random relative frequency $\boldsymbol{f}_{i j}$, a measurement error occurs:

$$
\boldsymbol{f}_{i j}=\boldsymbol{z}_{i j}+\boldsymbol{\eta}_{i j}
$$

Since each $\boldsymbol{t}_{i j}^{(k)} \boldsymbol{f}_{i j}^{(k)}(k=1, \ldots, 4)$ follows a binomial distribution conditional 
on $\boldsymbol{z}_{i j}$, we obtain

$$
\mathrm{E}\left(\boldsymbol{\eta}_{i j} \mid \boldsymbol{z}_{i j}\right)=\mathbf{0}, \quad \operatorname{var}\left(\boldsymbol{\eta}_{i j} \mid \boldsymbol{z}_{i j}\right)=\boldsymbol{\Delta}_{i j},
$$

where the diagonal elements of $\boldsymbol{\Delta}_{i j}$ are given by

$$
\boldsymbol{\Delta}_{i j}^{(k)}=\frac{1}{\boldsymbol{t}_{i j}^{(k)}} \boldsymbol{z}_{i j}^{(k)}\left(1-\boldsymbol{z}_{i j}^{(k)}\right) \quad(k=1, \ldots, 4) .
$$

We assume that the off-diagonal elements of $\boldsymbol{\Delta}_{i j}$ are zero, which is reasonable, because the components of $\boldsymbol{\eta}_{i j}$ represent "pure noise." In addition and for the same reason, we assume that $\mathrm{E}\left(\boldsymbol{\eta}_{j i} \mid \boldsymbol{z}_{i j}\right)=\mathbf{0}$ and that $\boldsymbol{\eta}_{i j}$ and $\boldsymbol{\eta}_{j i}$ are uncorrelated (conditional on $\boldsymbol{z}_{i j}$ and $\boldsymbol{z}_{j i}$ ).

\subsection{A model for $\boldsymbol{z}_{i j}$}

Since the four probabilities in $\boldsymbol{z}_{i j}$ will be correlated and, in addition, $\boldsymbol{z}_{i j}$ and $\boldsymbol{z}_{j i}$ will be correlated, we need to model an eight-dimensional distribution. The probabilities depend on the characteristics of the server and his or her opponent. Some of these characteristics are observable, some are not. An important characteristic is a player's "quality." In tennis a player's quality is partly measured by his or her official ranking. Although this is not a perfect measure of quality (for example, it does not account for "form of the day"), the ranking contains important information about the key probabilities, which we want to exploit.

Let $\mathrm{RANK}_{i}$ denote the ranking of player $i$ at the moment of the tournament. Direct use of the ranking is not satisfactory, because quality in tennis is a pyramid: the difference between the top two players (ranked 1 and 2) is generally larger than between two players ranked 101 and 102. As motivated in Klaassen and Magnus (2001), $\mathrm{RANK}_{i}$ is transformed into a smooth version of the "expected round" by defining

$$
r_{i}=8-\log _{2}\left(\mathrm{RANK}_{i}\right)
$$

For example, if $\mathrm{RANK}_{i}=4$ then $r_{i}=6.00$, and if $\mathrm{RANK}_{i}=3$ then $r_{i}=6.42$, indicating that both are expected to lose in round 6 (the semifinal in grand slam tournaments like Wimbledon). The additional 0.42 indicates that the number 3 ranked player is somewhat better than the player ranked 4 .

Apart from the ranking there are also quality components that we do not observe, such as form of the day, special ability on the court surface on which the match is played (grass for Wimbledon), and fear against a specific opponent. In addition, each player has his or her own style of play, 
a characteristic that may also affect the four key probabilities. None of these features is observed, but we correct for them by including two fourdimensional vectors $\varepsilon_{i j}$ and $\varepsilon_{j i}$ of unobservable effects.

We model the key probabilities in a given match between $i$ and $j$ as an eight-dimensional vector $\left(\boldsymbol{z}_{i j}^{\prime}, \boldsymbol{z}_{j i}^{\prime}\right)^{\prime}$, where

$$
\boldsymbol{z}_{i j}=\boldsymbol{\mu}+r_{i} \boldsymbol{\beta}_{S}+r_{j} \boldsymbol{\beta}_{R}+\boldsymbol{\varepsilon}_{i j} .
$$

The linearity assumption underlying this equation is acceptable when we use $r_{i}$ as a regressor, but not when we use $\mathrm{RANK}_{i}$, as preliminary nonparametric regressions show. The vectors $\boldsymbol{\beta}_{S}$ and $\boldsymbol{\beta}_{R}$ contain the effects of the server's and receiver's rankings on each probability in $\boldsymbol{z}_{i j}$, respectively. For the errors we assume

$$
\left(\begin{array}{l}
\varepsilon_{i j} \\
\varepsilon_{j i}
\end{array}\right) \sim\left(\left(\begin{array}{l}
\mathbf{0} \\
\mathbf{0}
\end{array}\right),\left(\begin{array}{cc}
\boldsymbol{\Sigma}_{1} & \boldsymbol{\Sigma}_{2} \\
\boldsymbol{\Sigma}_{2} & \boldsymbol{\Sigma}_{1}
\end{array}\right)\right)
$$

It is convenient to center $r_{i}$ and $r_{j}$. The zero mean standardization of the errors then implies that $\boldsymbol{\mu}$ is equal to the mean of $\boldsymbol{z}_{i j}$.

The assumption regarding the errors implies that $\varepsilon_{i j}$ is a random (instead of fixed) effect. Because the rankings $\mathrm{RANK}_{i}$ and $\mathrm{RANK}_{j}$ are determined before the tournament starts, we assume that there is no correlation between $\boldsymbol{\varepsilon}_{i j}$ and the rankings. To account for the correlations between the key probabilities for a given server we include a variance matrix $\boldsymbol{\Sigma}_{1}$. In addition, there may be correlations between the key probabilities across the two servers in a given match. For example, if player $i$ is better on the present court surface (grass, say) than his or her ranking indicates, or if player $i$ usually performs well against $j$, then especially the two last components of $\varepsilon_{i j}$ and $\varepsilon_{j i}$, which concern the winning probabilities, are negatively correlated. Thus we introduce a covariance matrix $\boldsymbol{\Sigma}_{2}$. Since the moments do not depend on the specific players $\left(i\right.$ and $j$ ), we have, for example, $\operatorname{cov}\left(\boldsymbol{\varepsilon}_{i j}^{(3)}, \boldsymbol{\varepsilon}_{j i}^{(4)}\right)=\operatorname{cov}\left(\boldsymbol{\varepsilon}_{j i}^{(3)}, \boldsymbol{\varepsilon}_{i j}^{(4)}\right)$, so that $\boldsymbol{\Sigma}_{2}$ is symmetric.

In summary, we have a model for the whole vector $\left(\boldsymbol{z}_{i j}^{\prime}, \boldsymbol{z}_{j i}^{\prime}\right)^{\prime}$ of probabilities that govern the two service series within a match. These probabilities are restricted to be constant within a match. However, in all other respects, the probabilities are unrestricted, as we allow for full heterogeneity across players and for possible correlations within a match. There are four free parameters in each of $\boldsymbol{\mu}, \boldsymbol{\beta}_{S}$, and $\boldsymbol{\beta}_{R}$, and ten free parameters in each of $\boldsymbol{\Sigma}_{1}$ and $\boldsymbol{\Sigma}_{2}$, in total 32 parameters.

\subsection{Moment conditions}

We employ the generalized method of moments (GMM) to estimate the 32 parameters. Hence we need to derive moment conditions, taking into account 
that the lengths of the service series across players vary, as do the lengths of the four service series for each player (in $\boldsymbol{t}_{i j}$ ). We define

$$
\boldsymbol{B}=\left(\boldsymbol{\mu}: \boldsymbol{\beta}_{S}: \boldsymbol{\beta}_{R}\right), \quad \boldsymbol{r}_{i j}^{\prime}=\left(1, r_{i}, r_{j}\right),
$$

and write (16) in matrix form as

$$
\boldsymbol{z}_{i j}=\boldsymbol{B} \boldsymbol{r}_{i j}+\boldsymbol{\varepsilon}_{i j}
$$

Combining (18) with (13) then gives

$$
\boldsymbol{f}_{i j}=\boldsymbol{B} \boldsymbol{r}_{i j}+\boldsymbol{\varepsilon}_{i j}+\boldsymbol{\eta}_{i j}
$$

where the fact that $\mathrm{E}\left(\boldsymbol{\eta}_{i j} \mid \boldsymbol{z}_{i j}\right)=\mathrm{E}\left(\boldsymbol{\eta}_{j i} \mid \boldsymbol{z}_{i j}\right)=\mathbf{0}$ implies that $\boldsymbol{\varepsilon}_{i j}$ is uncorrelated with $\boldsymbol{\eta}_{i j}$ and $\boldsymbol{\eta}_{j i}$. From the conditional moments (14) we find $\mathrm{E}\left(\boldsymbol{\eta}_{i j}\right)=\mathbf{0}$ and $\operatorname{var}\left(\boldsymbol{\eta}_{i j}\right)=\mathrm{E}\left(\boldsymbol{\Delta}_{i j}\right)$. Since $\boldsymbol{z}_{i j}$ (and hence $\left.\boldsymbol{\Delta}_{i j}\right)$ cannot be observed, we define the diagonal matrix $\widehat{\boldsymbol{\Delta}}_{i j}$ whose $k$-th diagonal element is given by

$$
\widehat{\boldsymbol{\Delta}}_{i j}^{(k)}=\frac{1}{\boldsymbol{t}_{i j}^{(k)}-1} \boldsymbol{f}_{i j}^{(k)}\left(1-\boldsymbol{f}_{i j}^{(k)}\right) \quad(k=1, \ldots, 4),
$$

and we note that $\mathrm{E}\left(\widehat{\boldsymbol{\Delta}}_{i j} \mid \boldsymbol{z}_{i j}\right)=\boldsymbol{\Delta}_{i j}$ so that $\mathrm{E}\left(\widehat{\boldsymbol{\Delta}}_{i j}\right)=\mathrm{E}\left(\boldsymbol{\Delta}_{i j}\right)$. (If $t_{i j}^{(k)}$ equals one or zero, then (19) breaks down. This does not occur in our data set, because the minimum number of second services in is 14 (men) and 5 (women), and the minimum number of first services in is 31 (men) en 14 (women).) The following expectations are then implied:

$$
\begin{aligned}
\mathrm{E}\left(\left(\boldsymbol{f}_{i j}-\boldsymbol{B} \boldsymbol{r}_{i j}\right) \boldsymbol{r}_{i j}^{\prime}\right) & =\mathrm{O} \\
\operatorname{var}\left(\boldsymbol{f}_{i j}-\boldsymbol{B} \boldsymbol{r}_{i j}\right) & =\boldsymbol{\Sigma}_{1}+\mathrm{E}\left(\widehat{\boldsymbol{\Delta}}_{i j}\right) \\
\operatorname{cov}\left(\boldsymbol{f}_{i j}-\boldsymbol{B} \boldsymbol{r}_{i j}, \boldsymbol{f}_{j i}-\boldsymbol{B} \boldsymbol{r}_{j i}\right) & =\boldsymbol{\Sigma}_{2} .
\end{aligned}
$$

The first moment is the usual OLS orthogonality condition (12 restrictions), the second moment concerns the within-server variance (10 restrictions, because of the symmetry), and the third moment captures the correlation between the frequency vectors of the two servers in a match (10 restrictions).

\subsection{Implementation}

Let $n$ denote the number of server series (twice the number of matches), the cross-section dimension of the panel. In our sample we have $n=516$ in the men's singles and $n=446$ in the women's singles. Because $n$ is substantially larger than the elements of $\boldsymbol{t}_{i j}$ (especially the elements that 
concern the second service), our asymptotic justification is based on large $n$ and finite $\boldsymbol{t}_{i j}$. The moment conditions have thus been set up in a format that is standard in studies that rely on large- $n$ asymptotics.

The set of moment conditions (20) contains 32 different elements. Each server $i$ in his or her match against $j$ has exactly one observation of each element. Let $\boldsymbol{m}_{i j}$ denote the 32-dimensional vector containing all observations:

$$
\boldsymbol{m}_{i j}=\left(\begin{array}{c}
\operatorname{vec}\left(\left(\boldsymbol{f}_{i j}-\boldsymbol{B} \boldsymbol{r}_{i j}\right) \boldsymbol{r}_{i j}^{\prime}\right) \\
\operatorname{vech}\left(\left(\boldsymbol{f}_{i j}-\boldsymbol{B} \boldsymbol{r}_{i j}\right)\left(\boldsymbol{f}_{i j}-\boldsymbol{B} \boldsymbol{r}_{i j}\right)^{\prime}-\boldsymbol{\Sigma}_{1}-\widehat{\boldsymbol{\Delta}}_{i j}\right) \\
\operatorname{vech}\left(\left(\boldsymbol{f}_{i j}-\boldsymbol{B} \boldsymbol{r}_{i j}\right)\left(\boldsymbol{f}_{j i}-\boldsymbol{B} \boldsymbol{r}_{j i}\right)^{\prime}-\boldsymbol{\Sigma}_{2}\right)
\end{array}\right)
$$

where vech() denotes the half-vec operator stacking the nonrepeated elements of a symmetric matrix. Obviously, $\mathrm{E}\left(\boldsymbol{m}_{i j}\right)=\mathbf{0}$ and one could use the sample average $(1 / n) \sum \boldsymbol{m}_{i j}$ in a GMM procedure to estimate the parameters. There are however two sources of inefficiency that we wish to take account of. First, all players serve a different number of points, so that the precision of the frequencies varies. Taking an unweighted average across players does not take this into account. Second, for a given server the number of points varies across the key frequencies. To increase the efficiency of our estimates, we weight each element of $\boldsymbol{m}_{i j}$ by the number of observations used to compute that element. Hence, we obtain a new moment vector

$$
\widetilde{\boldsymbol{m}}_{i j}=\boldsymbol{\Omega}_{i j} \boldsymbol{m}_{i j}
$$

where $\boldsymbol{\Omega}_{i j}$ is a diagonal $32 \times 32$ matrix with the 32 weights on the diagonal. This leads to the following GMM objective function:

$$
\min \left(\frac{1}{n} \sum \widetilde{\boldsymbol{m}}_{i j}\right)^{\prime} \boldsymbol{W}\left(\frac{1}{n} \sum \widetilde{\boldsymbol{m}}_{i j}\right),
$$

where we minimize over all 32 parameters. We use the standard optimal weighting matrix $\boldsymbol{W}$, that is, the inverse of the variance of the limiting distribution of $\widetilde{\boldsymbol{m}}_{i j}$. We begin with $\boldsymbol{W}=\boldsymbol{I}_{32}$ to obtain a consistent estimate of the parameter vector, then we estimate $\boldsymbol{W}$ by $\widehat{\boldsymbol{W}}$, and finally we minimize the objective function again using $\widehat{\boldsymbol{W}}$ instead of the identity matrix. This two-step procedure gives us consistent, asymptotically normal, and efficient estimates.

\subsection{Estimation results}

The estimates of $\boldsymbol{\mu}, \boldsymbol{\beta}_{S}, \boldsymbol{\beta}_{R}, \boldsymbol{\Sigma}_{1}$, and $\boldsymbol{\Sigma}_{2}$, together with their standard errors are reported in Table 2 . 


\section{TABLE 2}

The $\boldsymbol{\mu}$ parameters give the results for the average player and are estimated very precisely. On average $59.5 \%$ of the first services are in for the men (61.6\% for the women) and $86.4 \%$ of the second services (same for men and women). The similarity between men and women is remarkable. The scoring power of the service is of course rather different for men and women. If the first service is in then men score $74.0 \%$ (63.1\% for women) on average, and if the second service is in they score $59.4 \%$ ( $52.6 \%$ for women).

The $\boldsymbol{\beta}_{S}$-estimates show that the better a player is, the higher are the key probabilities. As expected, this is clearly true for $y_{1}$ and $y_{2}$ and (less strongly) also for $x_{1}$ and $x_{2}$. The estimates of $\boldsymbol{\beta}_{R}$ show just the opposite.

The estimates in the variance matrix $\boldsymbol{\Sigma}_{1}$ are presented as standard deviations (for the four diagonal elements) and correlations (for the six off-diagonal elements). Of the ten estimated parameters in the covariance matrix $\boldsymbol{\Sigma}_{2}$, seven are statistically and logically insignificant, and are set equal to zero. Yet we use 32 (rather than 25) moments at the estimation stage. This allows an overidentifying restrictions test (ORT), giving ORT $=11.77(0.11)$ for the men and ORT $=2.33(0.94)$ for the women ( $p$-values in brackets), thus providing further support for restricting the seven parameters to zero. The three remaining estimates in $\boldsymbol{\Sigma}_{2}$ are correlations, also the estimate indicated by $\left(y_{1}, y_{1}\right)$ (and similarly $\left.\left(y_{2}, y_{2}\right)\right)$ as it measures the correlation between $y_{1, i j}$ and $y_{1, j i}$. The three correlations are negative because if player $i$ is "in form" (relative to $j$ ) as a server he or she is also likely to be "in form" as a receiver.

The correlations in the (not reported) $25 \times 25$ variance matrix of the estimates are all small, so that the variables satisfy a high degree of orthogonality and there is no problem of multicollinearity. The estimates are all very plausible and the fact that all signs are the same for men and women underlines their significance.

\section{Identification of $\lambda$}

Now that we have estimated the distribution of $\left(\boldsymbol{z}_{i j}, \boldsymbol{z}_{j i}\right)$, we know the distribution of $\left(x_{1}, y_{1}\right)$ and $\left(x_{2}, y_{2}\right)$ for server $i$ in his/her match against $j$, but we do not yet know $\lambda$, a necessary ingredient for the estimation of the $y$ curve (12). The curvature parameter $\lambda$ is essentially unidentified, because the $y$-curve depends on three parameters and we only observe two points on each curve: $\left(x_{1}, y_{1}\right)$ and $\left(x_{2}, y_{2}\right)$. In principle we could increase the number of points on the curve by looking at sets instead of matches, but this does 
not help because the set results are too clustered.

Thus we treat $\lambda_{i j}$ as a random effect, in the same way as we have treated $\boldsymbol{\varepsilon}_{i j}$ in the estimation of $\boldsymbol{z}_{i j}$, and assume

$$
\lambda_{i j} \mid\left(\boldsymbol{z}_{i j}, \boldsymbol{z}_{j i}\right) \sim\left(\lambda, \sigma_{\lambda}^{2}\right) .
$$

We shall obtain "conservative" estimates of $\lambda$ and $\sigma_{\lambda}$, in line with our discussion in Section 2.3, in the sense that we shall solve

$$
\max _{\lambda, \sigma_{\lambda}} \frac{1}{n} \sum \mathrm{E}\left(\mathrm{eff}_{i j}\right)
$$

where eff $=p / p^{*}$ is the efficiency defined in (5), and $n=1016$ denotes the number of players. We maximize the average of the expected efficiency (over the $n$ players), because the elements over which we maximize are all positive, so that the average is equal to the mean absolute error and therefore takes account of the spread as well as the location. This measure is therefore preferable over the median (only location).

The (nontrivial) estimation procedure is described below. We shall see that the conservative estimate of $\sigma_{\lambda}$ is essentially zero, so that we may take $\lambda_{i j}=\lambda$ (constant). Apparently we may assume that for each player the $y$-curve has the same curvature (but different height and slope).

We treat men and women separately. For both men's singles and women's singles we consider 508 matches, that is, all singles matches played at Wimbledon during 1992-1995. For each match we require the rankings $r_{i}$ and $r_{j}$, but not the summary statistics used in Section 4 .

\subsection{Monte Carlo}

In Section 4 we estimated the 25 parameters in $\boldsymbol{\mu}, \boldsymbol{\beta}_{S}, \boldsymbol{\beta}_{R}, \boldsymbol{\Sigma}_{1}$, and $\boldsymbol{\Sigma}_{2}$, and also the $25 \times 25$ variance matrix. We collect the 25 parameters in a vector $\boldsymbol{\theta}$, denote its estimator by $\widehat{\boldsymbol{\theta}}$, the asymptotic variance matrix of $\widehat{\boldsymbol{\theta}}$ by $\boldsymbol{V}$, and its estimator by $\widehat{\boldsymbol{V}}$. Then, approximately, $\widehat{\boldsymbol{\theta}} \sim \mathrm{N}(\boldsymbol{\theta}, \boldsymbol{V})$.

The Monte Carlo procedure consists of two stages. In the first stage we draw, for $r=1, \ldots, R(R=50)$, a vector $\boldsymbol{\theta}^{(r)}$ from the $\mathrm{N}(\widehat{\boldsymbol{\theta}}, \widehat{\boldsymbol{V}})$ distribution under the restriction that the matrix

$$
\left(\begin{array}{cc}
\boldsymbol{\Sigma}_{1}^{(r)} & \boldsymbol{\Sigma}_{2}^{(r)} \\
\boldsymbol{\Sigma}_{2}^{(r)} & \boldsymbol{\Sigma}_{1}^{(r)}
\end{array}\right)
$$

is positive definite. Hence we draw from a truncated normal distribution. The first stage of the Monte Carlo draw is independent of $\lambda_{i j}$. 
In the second stage we simulate $\mathrm{E}\left(\mathrm{eff}_{i j}\right)$ by drawing $\left(\boldsymbol{z}_{i j}, \boldsymbol{z}_{j i}, \lambda_{i j}, \lambda_{j i}\right)$, for each of the 508 matches and for each of the $R$ draws of $\boldsymbol{\theta}$. By (16) and (17) we have in each match

$$
\left(\begin{array}{c}
\boldsymbol{z}_{i j} \\
\boldsymbol{z}_{j i}
\end{array}\right) \sim\left(\left(\begin{array}{c}
\boldsymbol{\mu}^{(r)}+r_{i} \boldsymbol{\beta}_{S}^{(r)}+r_{j} \boldsymbol{\beta}_{R}^{(r)} \\
\boldsymbol{\mu}^{(r)}+r_{j} \boldsymbol{\beta}_{S}^{(r)}+r_{i} \boldsymbol{\beta}_{R}^{(r)}
\end{array}\right),\left(\begin{array}{cc}
\boldsymbol{\Sigma}_{1}^{(r)} & \boldsymbol{\Sigma}_{2}^{(r)} \\
\boldsymbol{\Sigma}_{2}^{(r)} & \boldsymbol{\Sigma}_{1}^{(r)}
\end{array}\right)\right) .
$$

Since the densities of the four frequencies in $\boldsymbol{f}_{i j}$ have the appearance of normal curves, it seems reasonable to assume normality of the distribution of $\left(\boldsymbol{z}_{i j}, \boldsymbol{z}_{j i}\right)$, but truncated for two reasons. First, we must have $x_{0} \leq x_{1} \leq 1$ and $x_{0} \leq x_{2} \leq 1$. In particular, the condition $x_{2} \leq 1$ must be imposed. Second, conditions R1 and R2 must hold, which is the case if and only if condition (7) holds. Using (11), the truncation thus depends on $\lambda_{i j}$ because

$$
1 \leq \frac{y_{1, i j} x_{2, i j}^{\lambda_{i j}}-y_{2, i j} x_{1, i j}^{\lambda_{i j}}}{y_{1, i j}-y_{2, i j}} \leq \frac{x_{2, i j}^{\lambda_{i j}}-x_{1, i j}^{\lambda_{i j}}}{y_{1, i j}-y_{2, i j}}+x_{0}^{\lambda_{i j}},
$$

where we set $x_{0}=0.4$. We also assume normality of $\left(\lambda_{i j}, \lambda_{j i}\right)$, but truncated because of the restriction $\lambda>0$. We now draw $S=50$ ten-dimensional vectors of independent realizations $\left(\boldsymbol{u}_{i j}, \boldsymbol{u}_{j i}\right)$ from the uniform distribution defined on the $[0,1]$ interval. We transform them into $S$ draws $\left(\boldsymbol{z}_{i j}, \boldsymbol{z}_{j i}, \lambda_{i j}, \lambda_{j i}\right)$ using the GHK procedure; see Hajivassiliou, McFadden and Ruud (1996). The resulting draws will satisfy regularity conditions $\mathrm{R} 1$ and $\mathrm{R} 2$, have positive $\lambda$, and all probabilities will be in the $[0,1]$ interval. We then compute $\operatorname{eff}_{i j}$ for each draw, and approximate $\mathrm{E}\left(\mathrm{eff}_{i j}\right)$ by taking the average over the $S$ draws.

\subsection{Conservative estimation of $\lambda$}

The result of the two-stage procedure is that we can calculate, for each of the $R$ draws $\boldsymbol{\theta}^{(r)}$ and for each server, the expectation $\mathrm{E}\left(\mathrm{eff}_{i j}\right)$ as a function of $\lambda$ and $\sigma_{\lambda}$, and hence also the average expected efficiency

$$
\overline{\operatorname{eff}}\left(\lambda, \sigma_{\lambda}\right):=\frac{1}{n} \sum \mathrm{E}\left(\operatorname{eff}_{i j}\right)
$$

To estimate $\lambda$ and $\sigma_{\lambda}$ conservatively, we calculate the function $\overline{\operatorname{eff}}\left(\lambda, \sigma_{\lambda}\right)$ for different values of $\lambda$ and $\sigma_{\lambda}$, where in each function calculation the draw of $\left(\boldsymbol{z}_{i j}, \boldsymbol{z}_{j i}, \lambda_{i j}, \lambda_{j i}\right)$ is based on the same draw of $\left(\boldsymbol{u}_{i j}, \boldsymbol{u}_{j i}\right)$. Because of the concavity of the underlying functions, the function $\overline{\operatorname{eff}}\left(\lambda, \sigma_{\lambda}\right)$ will have a maximum in a suitably chosen parameter range.

The $R$ maximizing parameter values have a mean (standard error) of $\widehat{\lambda}=3.07(0.13)$ and $\widehat{\sigma}_{\lambda}=0.002(0.003)$ for the men, and $\widehat{\lambda}=3.77(0.35)$ and 
$\widehat{\sigma}_{\lambda}=0.005(0.008)$ for the women. Since $\widehat{\sigma}_{\lambda}$ is not significantly different from zero, we set $\sigma_{\lambda}=0$ and re-estimate $\lambda$. The $R$ draws for $\lambda_{\text {con }}$ have a mean (standard error) of 3.07 (0.13) in the men's singles and $3.83(0.37)$ in the women's singles, where the subscript "con" is a reminder of the fact that we estimate $\lambda$ conservatively in the sense that $\lambda_{\text {con }}$ provides an average expected efficiency which is at least as high as the true average expected efficiency. (If we maximize the median instead of the mean of the expected efficiency, we obtain $\lambda_{\text {con }}=3.06$ (0.13) for the men and $\lambda_{\text {con }}=3.71$ (0.35) for the women.)

The estimate of $\lambda$ is significantly different for men and women, and is rather precise. There is thus ample evidence that the $y$-curve is concave $(\lambda>1)$ in the relevant interval. At the end of this admittedly complicated estimation procedure we thus have, for each of the $R$ draws of $\boldsymbol{\theta}, S$ feasible $8 \times 1$ vectors $\left(\boldsymbol{z}_{i j}, \boldsymbol{z}_{j i}\right)$ for all 508 matches and one $\lambda_{\text {con }}$. In short, we have $S=50$ feasible $9 \times 1$ vectors $\left(\boldsymbol{z}_{i j}, \boldsymbol{z}_{j i}, \lambda_{\text {con }}\right)$.

\subsection{Sensitivity}

A crucial element in this paper is the specification of the $y$-curve and the estimation of its curvature. It is therefore important to find out how sensitive our results are to (small) deviations from the chosen specification.

\begin{tabular}{cccc}
\multicolumn{4}{c}{ Table 3-Estimates of $\lambda_{\text {con }}$} \\
\hline \hline$y$-curve & Curvature & Men & Women \\
\hline$C_{0}$ & $\lambda$ & 3.0677 & 3.7727 \\
& & $(0.1315)$ & $(0.3455)$ \\
$C_{1}$ & $\lambda$ & 3.0553 & 3.4338 \\
& & $(0.1246)$ & $(0.2751)$ \\
$C_{2}$ & $\lambda_{1}$ & 3.1373 & 3.5720 \\
& & $(0.1879)$ & $(0.2816)$ \\
& $\lambda_{2}$ & 2.8955 & 2.9366 \\
& & $(0.2538)$ & $(0.6754)$ \\
\hline
\end{tabular}

In Table 3 we compare the conservative $\lambda$-estimates from three different specifications of the $y$-curve. The curve $C_{0}$ is our preferred specification (6), while the curves $C_{1}$ and $C_{2}$ are based on the idea that the most important aspect of the $y$-curve is the area around $x_{1}$ and $x_{2}$. Suppose we allow two $y$-curves: one around $x_{1}$ and one around $x_{2}$. The two curves are both power curves like (6), but with different sets of parameters $(\alpha, \tau, \lambda)$. In the curve $C_{1}$ we set $\lambda_{1}=\lambda_{2}$, while in the curve $C_{2}$ we do not restrict $\lambda$. We see that for the men the conservative estimate of $\lambda$ is hardly affected; the value $\lambda_{\text {con }}=3.1$ 
appears to be very stable. For the women slightly less so. Still, there is no statistical support for rejecting the specification (6).

\section{$6 \quad$ Efficiency results}

For both men and women the previous section provides $S$ feasible $9 \times 1$ vectors $\left(\boldsymbol{z}_{i j}, \boldsymbol{z}_{j i}, \lambda_{\text {con }}\right)$ for each of the 508 matches (1016 players). Thus we obtain a distribution of the $50 \times 1016=50,800$ observations on the efficiency eff $_{i j}$. The density is estimated nonparametrically using the quartic kernel. This
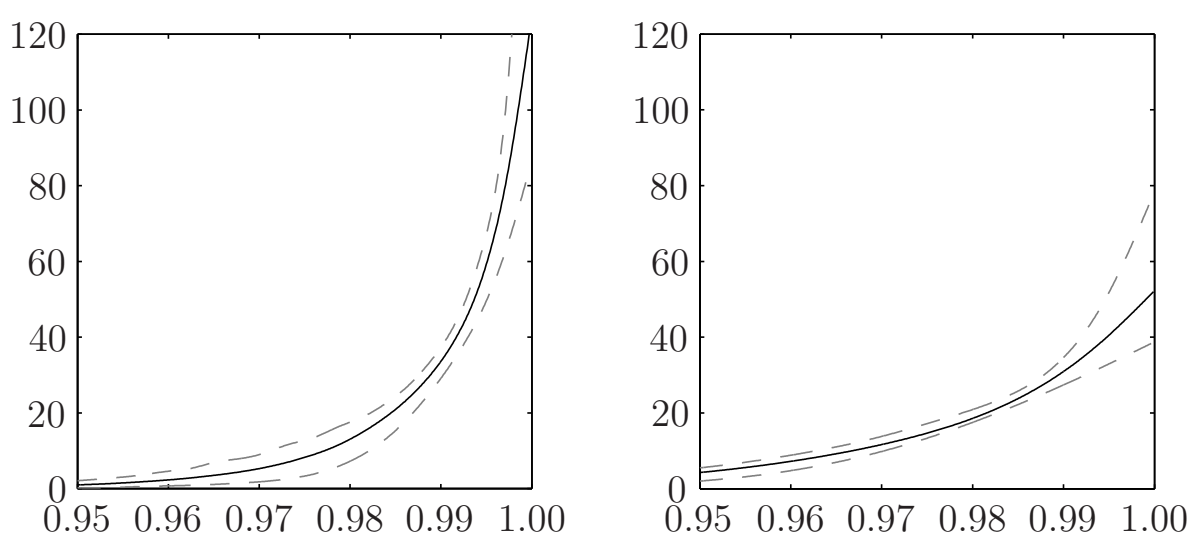

Figure 2. Distribution of the efficiency $p / p^{*}$ across players (men left, women right).

standard approach, however, leads to a downward bias near the boundary of the support, in our case near one. To avoid the boundary effect we use a local linear fitting method, described in Karunamuni and Alberts (2005). Repeating this for all $R$ replications we find the median and the 2.5 and 97.5 percentiles in Figure 2. The $95 \%$ band reflects uncertainty resulting from the GMM procedure of Section 4.

For the men, the mean of the (median) distribution is $98.9 \%$ (with a standard error of $0.2 \%$ ) and the distribution can be characterized by the $5 \%$, $25 \%, 50 \%, 75 \%$, and $95 \%$ quantiles given by $(96.7,98.6,99.3,99.7,99.9)$. Hence, on average the inefficiency is $1.1 \%$, while $25 \%$ of the players have an inefficiency of more than $1.4 \%$ and $5 \%$ of the players an inefficiency of more than $3.3 \%$.

For the women, the mean of the distribution is $98.0 \%(0.3 \%)$ and the $5 \%$, $25 \%, 50 \%, 75 \%$, and $95 \%$ quantiles are given by $(94.2,97.2,98.6,99.4,99.8)$. Hence, on average the inefficiency is $2.0 \%$, while $25 \%$ of the players have an 
inefficiency of more than $2.8 \%$ and $5 \%$ of the players an inefficiency of more than $5.8 \%$. The women are thus less efficient servers than the men.

If we assume, instead of model $C_{0}$, one of the models $C_{1}$ or $C_{2}$, then $\lambda_{\text {con }}$ changes little (see Table 3 ), but this does not necessarily imply that the efficiency $p / p^{*}$ also changes little. This, however, is the case. For example, under model $C_{2}$ the median efficiency is estimated as $99.0 \%$ for men $(89.9 \%$ under $\left.C_{0}\right)$ and $98.1 \%$ for women $\left(98.0 \%\right.$ under $\left.C_{0}\right)$. The (in)efficiency estimates are therefore not very sensitive to small misspecifications in the $y$-curve.

We reject the hypothesis that top tennis players use an efficient service strategy and we obtain a lower bound of the estimated mean inefficiency in tennis: $1.1 \%$ for men and $2.0 \%$ for women. The estimated inefficiencies may seem small, but (a) the estimates are lower bounds so that the actual inefficiencies will be larger, and (b) the inefficiencies are calculated at point level.

We can also calculate the inefficiency at game-, set-, and match-level. If we consider a game, the inefficiency increases, not because the players perform differently but because of the structure of the tennis scoring system: from $1.1 \%$ at point level to $1.4 \%$ at game level for the men, and from $2.0 \%$ at point level to $4.0 \%$ at game level for the women. The inefficiency increases further if we consider a match (perhaps the most natural unit) between two players $i$ and $j$. The (in)efficiency now does not only depend on the efficiency of player $i$ but also of player $j$. What would be the efficiency gain for player $i$ if he or she serves efficiently while player $j$ does not? This question is answered in Figure 3. For all $S$ draws of $\left(\boldsymbol{z}_{i j}, \boldsymbol{z}_{j i}, \lambda_{c o n}\right)$ and for each match,
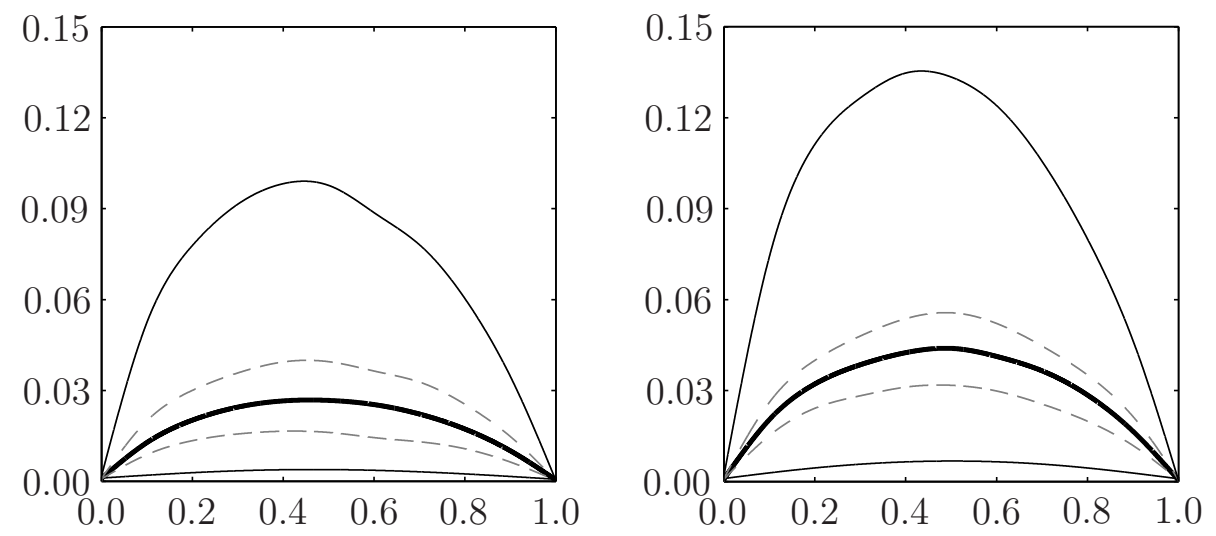

Figure 3. Efficiency gain $m^{*}-m$ as a function of $m$, match level (men left, women right).

we compute the probabilities $m_{i j}$ ( $i$ wins the match against $j$, both serving 
normally) and $m_{i j}^{*}$ ( $i$ wins the match against $j, i$ serves optimally, $j$ serves normally). Then we regress $\left(m_{i j}^{*}-m_{i j}\right)$ on $m_{i j}$ (50,800 observations) using nonparametric quantile regressions. We use the same kernel as above, including the boundary correction method, though now not only for $m_{i j}$ near one but also near zero. The fact that the support is bounded also implies that the fitted regression curve exhibits flattening at the boundaries. We avoid this by using the local linear regression method instead of the more standard locally weighted averaging approach; see Fox (2000). Finally, to account for the skewness of $m_{i j}^{*}-m_{i j}$, we use quantile regression instead of the usual (mean) regression, taking the 10, 50 and $90 \%$ quantiles; see Koenker $(2005$, p. 222) on locally linear quantile regression. This whole procedure gives median and 10 and 90 percentiles regression curves, where the $80 \%$ band represents the variation of $m_{i j}^{*}-m_{i j}$ across servers. These are the three solid lines in Figure 3. We repeat the procedure for each of the $R$ replications, so that we can estimate the $95 \%$ confidence intervals around the three curves to measure the impact of the GMM estimation uncertainty. For simplicity, Figure 3 only contains the confidence band (dashed) around the median curve. If the two players are approximately of equal strength then the median efficiency gain at match level for the efficient server is $2.7 \%$ for the men and $4.4 \%$ for the women. Moreover, $10 \%$ of the players will have an efficiency increase of more than 10-15\%.

We now have estimates of the inefficiency at point level (1.1\%), game level $(1.4 \%)$, and match level (2.7\% against a player of equal strength) for the men $(2.0 \%, 4.0 \%$, and $4.4 \%$ for the women), but this does not yet answer the question what the effect of inefficiency is. To answer this question we run a hypothetical tournament of 128 players (seven rounds, like Wimbledon), where in each match both players have probability 0.50 to win the match, except one player who serves efficiently. The only efficient player has $52.7 \%$ (that is, an additional $2.7 \%$ ) probability of winning a match in the men's singles (54.4\% in the women's singles). What is the expected monetary gain for the efficient player? In grand slam tournaments the paycheck approximately doubles in each round. If we assume that this is exactly true, then the expected paycheck for the efficient player will rise by $18.7 \%$ for men and $32.8 \%$ for women. At Wimbledon this would mean an expected additional income of approximately $\$ 10,000$ for the efficient man and $\$ 15,000$ for the efficient woman. Hence, even though the inefficiency at point level may seem small, the monetary effect of inefficiency is not small at all.

Since a player has two services, we may ask whether an optimal first service is more or less important than an optimal second service. This question is answered in Figure 4, where we only consider the median. The graph $\left(x_{1}^{*}, x_{2}^{*}\right)$ is the same as the median in Figure 3, but now decomposed into 

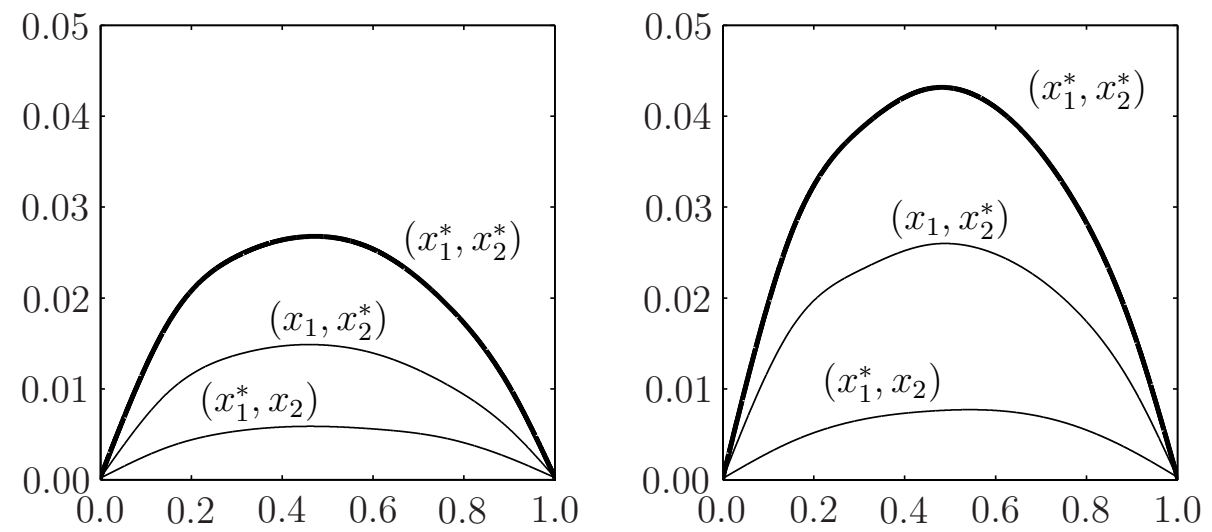

Figure 4. Decomposition of efficiency gain into first and second service, match level (men left, women right).

$\left(x_{1}, x_{2}^{*}\right)$ where only the second service is optimal and $\left(x_{1}^{*}, x_{2}\right)$ where only the first service is optimal. The figure shows that players can achieve a larger efficiency gain on their second service than on their first service, possibly because of a misguided (inefficient) fear for a double fault.

Finally we ask whether better players are more efficient than weaker players, as we would perhaps expect. This is no tautology because better players could also be more talented and equally efficient as weaker players. Our basic equality is

$$
\text { success }=\text { talent }+ \text { efficiency } .
$$

A talented but not so efficient player may be as successful as a less talented player who is more efficient. We observe success by the ranking of a player, and we can measure efficiency; talent is unobserved, but can be deduced.

We run the following simple linear regression:

$$
\operatorname{eff}_{i j}=\beta_{1}+\beta_{2} r_{i}+\beta_{3}\left|r_{i}-r_{j}\right|+\beta_{4} r_{i} r_{j}+\xi_{i j} .
$$

To account for the random effects in eff $i j$, we use for each server the draws $\left(\boldsymbol{z}_{i j}, \lambda_{\text {con }}\right)$ from Section 5.2 , so that we have 50,800 observations. The regression is performed for each of the $R$ replications of $\boldsymbol{\theta}$. To combine the results across $\boldsymbol{\theta}$, we draw 100 times from each estimated distribution of $\widehat{\boldsymbol{\beta}}$ and, to account for skewness, the resulting 5000 draws are summarized in a $95 \%$ confidence interval around the median.

Two conclusions emerge. First, the confidence interval for $\widehat{\beta}_{2}$ is $(0.0004$, $0.0015)$ for the men and $(0.0004,0.0023)$ for the women. Hence, higherranked players are indeed more efficient. Second, the confidence interval for 
$\widehat{\beta}_{3}$ is $(-0.0010,-0.0001)$ for the men and $(-0.0011,0.0000)$ for the women. Hence, the closer the contest, the more efficient players are forced to be.

\section{Conclusions and implications}

In a tennis match between two players, the objective of each player is to win the match. If it is a match between two amateurs (possibly business partners), one player may be hesitant to beat his or her boss or to win too decisively. But top professionals do not act like this. If we were studying a minor tournament, even a top player might not fully commit because he or she wants to be fit for next week's major tournament. That is why we study only a major tournament, namely Wimbledon. Our "utility" function to be maximized is the probability that a player (given his or her opponent and given the strengths of both players) wins a point while serving. It seems likely that this is indeed the function which players wish to maximize.

We asked the question whether the service strategy for top tennis players, playing in a top tournament, is efficient. The answer is that it is not. Our estimates are "conservative" and thus provide lower bounds - the real inefficiencies are larger. Our model and set-up are very general. Most assumptions have been subjected to extensive sensitivity analyses in order to check whether (small) deviations from the assumptions have a significant impact on the results. We are confident that we have proved inefficiency among top players. In addition, we have obtained estimates for the lower bound of the inefficiency.

The chosen level of investigation in this paper is a point. At point level the inefficiency is on average $1.1 \%$ for men (2.0\% for women). At game level the inefficiency is $1.4 \%$ (4.0\%), and at match level, assuming two players of the same strength, $2.7 \%$ (4.4\%). These differences do not reflect the players but the scoring system. In terms of expected monetary gains we have calculated that the expected paycheck for the efficient player will rise by $18.7 \%$ for men and $32.8 \%$ for women.

What is the reason for this inefficiency? Perhaps top tennis players know their $y$-curve, but are not able to solve the optimization problem. Or do they correctly solve the optimization, but on the wrong $y$-curve? The decomposition into first and second service (Figure 4) may help us here. From the point of view of achieving optimality it is much easier for a server to work out the optimal second service (maximize $w(x)$ ) than to work out the optimal first service (maximize $w(x)+(1-x) w\left(x_{2}^{*}\right)$ ). Nevertheless, the second service appears to be less efficient than the first service. This provides evidence that, although players may be maximizers, they do not maximize the correct 
function.

We also find that higher-ranked players are more efficient than lowerranked players, and that the closer the match, the more efficient a player serves.

Let us now try and relate these results to economics, and in particular to our original question whether economic agents are successful maximizers. First, our results show that economic agents and even top agents are not fully efficient, and that this inefficiency can be financially nontrivial. Second, the different inefficiency results at point-, game-, and match level show that the market form has an impact on the measurement of inefficiency. For example, in the case of Bertrand competition for homogeneous goods, a "winner-takesall" effect is induced, and this nonlinearity is not related to productivity but to the nature of the competition. Third, the fact that tighter matches cause players to be more efficient suggests that in a more competitive market firms are forced to be more efficient; otherwise they will be driven out of the market. This supports the view of many policy makers that measures aimed at strengthening market mechanisms lead to a more efficient economy.

What contribution, if any, does this paper make to the closely related subject of rational behavior? In rational choice theory the "rational man" is assumed to (a) know his or her preferences over all relevant alternatives, and (b) choose the best alternative. In our tennis framework this corresponds to (a) a player who knows the pay-off structure $p\left(x_{1}, x_{2}\right)$, and (b) is able to maximize that pay-off.

We find inefficiency and hence the agent does not know the pay-off structure and/or is not able to solve the maximization problem. This would reject rationality in favor of bounded rationality, and therefore corroborates findings from the experimental economics literature.

The inefficiency is larger for the second than for the first service, even though the maximization problem for the second service is easier. This suggests that at least (a) is rejected, that is, the agent does not fully use his or her true preference relations.

The inefficiency is small and hence rationality might be an acceptable approximation in many applications, at least for the top agents. Friedman's (1953, p. 21) expert billiard players are like our top tennis players: they make their shots as if they know and can implement the complicated mathematical formulas underlying the optimal paths of the balls. This will be approximately true for expert billiard players, but not necessarily for the average billiard player. Since we find that the efficiency is smaller for weak players than for strong players, rationality may not be a good approximation for the average tennis player, the average billiard player, or the average 
economic agent. Hence, in spite of Friedman's (p. 21) assertion that

It is only a short step from these examples to the economic hypothesis that under a wide range of circumstances individual firms behave as if they were seeking rationally to maximize their expected returns,

it is not justified to think of "individual firms" (that is, non-top firms) as rational.

\section{Acknowledgements}

We thank IBM UK and The All England Club at Wimbledon for their kindness in providing the data. We also thank Jan Boone, Tijmen Daniëls, Bas Donkers, Noud van Giersbergen, Siem Jan Koopman, Knox Lovell, Thijs ten Raa, and Mark Steel for help and constructive discussions.

\section{References}

Carlton, Dennis W.; Frankel, Alan S. and Landes, Elisabeth M. "The Control of Externalities in Sports Leagues: An Analysis of Restrictions in the National Hockey League." Journal of Political Economy, 2004, 112(1), pp. S268-S288.

Charnes, A.; Cooper, W.W. and Rhodes, E. "Measuring the Efficiency of Decision-Making Units." European Journal of Operational Research, 1978, 2(6), pp. 429-444.

Chiappori, P.-A.; Levitt, S. and Groseclose, T. "Testing Mixed-Strategy Equilibria When Players are Heterogeneous: The Case of Penalty Kicks in Soccer." American Economic Review, September 2002, 92(4), pp. 1138 1151 .

Dixon, Mark J. and Coles, Stuart G. "Modelling Association Football Scores and Inefficiencies in the Football Betting Market." Applied Statistics, 1997, 46(2), pp. 265-280.

Ehrenberg, Ronald G. and Bognanno, Michael L. "Do Tournaments Have Incentive Effects?" Journal of Political Economy, December 1990, 98(6), pp. 1307-1324. 
Førsund, Finn R.; Lovell, C.A. Knox and Schmidt, Peter. "A Survey of Frontier Production Functions and of Their Relationship to Efficiency Measurement." Journal of Econometrics, 1980, 13(1), pp. 5-25.

Fox, John. Nonparametric Simple Regression: Smoothing Scatterplots. Thousand Oaks CA: Sage Publications, 2000.

Friedman, Milton. Essays in Positive Economics. Chicago: Chicago University Press, 1953.

Gale, D. "Optimal Strategy for Serving in Tennis." Mathematics Magazine, 1971, 44, pp. 197-199.

Gandar, John; Zuber, Richard; O'Brian, Thomas and Russo, Ben. "Testing Rationality in the Point Spread Betting Market." The Journal of Finance, September 1988, 43(4), pp. 995-1008.

Garicano, Luis; Palacios-Huerta, Ignacio and Prendergast, Canice. "Favoritism under Social Pressure." The Review of Economics and Statistics, May 2005, 87(2), pp. 208-216.

Goff, Brian L; McCormick, Robert E. and Tollison, Robert, D. "Racial Integration as an Innovation; Empirical Evidence from Sports Leagues." American Economic Review, March 2002, 92(1), pp. 16-26.

Golec, Joseph and Tamarkin, Maurry. "The Degree of Inefficiency in the Football Betting Market." Journal of Financial Economics, 1991, 30, pp. 311-323.

Gray, Philip K. and Gray, Stephen F. "Testing Market Efficiency: Evidence from the NFL Sports Betting Market." The Journal of Finance, September 1997, 52(4), pp. 1725-1737.

Gwartney, James and Haworth, Charles. "Employer Costs and Discrimination: The Case of Baseball." Journal of Political Economy, JulyAugust 1974, 82(4), pp. 873-881.

Hajivassiliou, Vassilis; McFadden, Daniel and Ruud, Paul. "Simulation of Multivariate Normal Rectangle Probabilities and Their Derivatives Theoretical and Computational Results." Journal of Econometrics, 1996, 72, pp. 85-134.

Julien, Bruno and Salanié, Bernard. "Estimating Preferences under Risk: The Case of Racetrack Bettors." Journal of Political Economy, 2000, 108(3), pp. 503-530. 
Kahn, Lawrence M. and Sherer, Peter D. "Racial Differences in Professional Basketball Players' Compensation." Journal of Labor Economics, January 1988, 6(1), pp. 40-61.

Kanazawa, Mark T. and Funk, Jonas P. "Racial Discrimination in Professional Basketball: Evidence from Nielsen Ratings. Economic Inquiry, October 2001, 39(4), pp. 599-608.

Karunamuni, Rohana J. and Alberts, Tom. "A Generalized Reflection Method of Boundary Correction in Kernel Density Estimation." The Canadian Journal of Statistics, 2005, 33(4), pp. 497-509.

Klaassen Franc J.G.M. and Magnus, Jan R. "Are Points in Tennis Independent and Identically Distributed? Evidence from a Dynamic Binary Panel Data Model." Journal of the American Statistical Association, 2001, 96, pp. 500-509.

Koenker, Roger. Quantile Regression. Econometric Society Monographs no. 38, New York: Cambridge University Press, 2005.

Koop, Gary; Osiewalsky, Jacek and Steel, Mark F.J. "Bayesian Efficiency Analysis through Individual Effects: Hospital Cost Frontiers." Journal of Econometrics, 1997, 76, pp. 77-105.

Kumbhakar, Subal C. and Lovell, C.A. Knox. Stochastic Frontier Analysis. New York: Cambridge University Press, 2000.

Lazear, Edward P. and Rosen, Sherwin. "Rank-Order Tournaments as Optimum Labor Contracts." Journal of Political Economy, 1981, 89(5), pp. 841-864.

Leibenstein, Harvey. "Allocative Efficiency vs. 'X-Efficiency'." American Economic Review, June 1966, 56(2), pp. 392-415.

Levitt, Steven D. "Why Are Gambling Markets Organised so Differently from Financial Markets?" The Economic Journal, April 2004, 114, pp. 223-246.

Magnus, Jan R. and Klaassen Franc J.G.M. "On the Advantage of Serving First in a Tennis Set: Four Years at Wimbledon," The Statistician (Journal of the Royal Statistical Society, Series D), 1999a, 48, pp. 247256. 
Magnus, Jan R. and Klaassen Franc J.G.M. "The Effect of New Balls in Tennis: Four Years at Wimbledon," The Statistician (Journal of the Royal Statistical Society, Series D), 1999b, 48, pp. 239-246.

Nardinelli, Clark and Simon, Curtis. "Customer Racial Discrimination in the Market for Memorabilia: The Case of Baseball." The Quarterly Journal of Economics, August 1990, 105(3), pp. 575-595.

Sauer, Raymond D.; Brajer, Vic; Ferris, Stephen P. and Marr, M. Wayne. "Hold Your Bets: Another Look at the Efficiency of the Gambling Market for National Football League Games." Journal of Political Economy, February 1988, 96(1), pp. 206-231.

Schmidt, Martin B. and Berri, David J. "The Impact of Labor Strikes on Consumer Demand: An Application to Professional Sports." American Economic Review, March 2004, 94(1), pp. 344-357.

Schmidt, Peter. "Frontier Production Functions." Econometric Reviews, 1985-1986, 4(2), pp. 289-328.

Stigler, George J. "The Xistence of X-Efficiency." American Economic Review, March 1976, 66(1), pp. 213-216.

Stone, Eric W. and Warren Jr, Ronald S. "Customer Discrimination in Professional Basketball: Evidence from the Trading-Card Market." Applied Economics, 1999, 31, pp. 679-685.

Szymanski, Stefan. "A Market Test for Discrimination in the English Professional Soccer Leagues." Journal of Political Economy, 2000, 108(3), pp. 590-603.

Walker, Mark and Wooders, John. "Minimax Play at Wimbledon." American Economic Review, December 2001, 91(5), pp. 1521-1538.

Zuber, Richard A.; Gandar, John M. and Bowers, Benny D. "Beating the Spread: Testing the Efficiency of the Gambling Market for National Football League Games." Journal of Political Economy, August 1985, 93(4), pp. 800-806. 
Table 2-Estimates of Men's and Women's Singles Equations (16) and (17)

\begin{tabular}{lcrcrc}
\hline \hline & & \multicolumn{2}{c}{ Men } & \multicolumn{2}{c}{ Women } \\
\cline { 2 - 6 } Coefficients & Estimate & Standard error & Estimate & Standard error \\
\hline $\boldsymbol{\mu}$ & $x_{1}$ & 0.5947 & 0.0034 & 0.6157 & 0.0051 \\
& $x_{2}$ & 0.8642 & 0.0031 & 0.8644 & 0.0047 \\
& $y_{1}$ & 0.7403 & 0.0037 & 0.6308 & 0.0051 \\
& $y_{2}$ & 0.5942 & 0.0044 & 0.5262 & 0.0077 \\
\hline $\boldsymbol{\beta}_{S}$ & $x_{1}$ & 0.0010 & 0.0015 & 0.0056 & 0.0029 \\
& $x_{2}$ & 0.0028 & 0.0013 & 0.0108 & 0.0031 \\
& $y_{1}$ & 0.0148 & 0.0017 & 0.0228 & 0.0025 \\
& $y_{2}$ & 0.0110 & 0.0020 & 0.0144 & 0.0037 \\
\hline $\boldsymbol{\beta}_{R}$ & $x_{1}$ & 0.0007 & 0.0015 & 0.0042 & 0.0024 \\
& $x_{2}$ & -0.0011 & 0.0013 & -0.0002 & 0.0025 \\
& $y_{1}$ & -0.0062 & 0.0017 & -0.0198 & 0.0026 \\
& $y_{2}$ & -0.0088 & 0.0021 & -0.0200 & 0.0039 \\
\hline $\boldsymbol{\Sigma}_{1}$ & $x_{1}$ & 0.0488 & 0.0049 & 0.0685 & 0.0059 \\
& $x_{2}$ & 0.0317 & 0.0051 & 0.0505 & 0.0075 \\
& $y_{1}$ & 0.0547 & 0.0042 & 0.0486 & 0.0083 \\
& $y_{2}$ & 0.0540 & 0.0062 & 0.0754 & 0.0113 \\
& $\left(x_{2}, x_{1}\right)$ & 0.6390 & 0.1540 & 0.2973 & 0.1343 \\
& $\left(y_{1}, x_{1}\right)$ & -0.0985 & 0.1226 & -0.5923 & 0.1720 \\
& $\left(y_{2}, x_{1}\right)$ & 0.0652 & 0.1377 & 0.2415 & 0.1782 \\
& $\left(y_{1}, x_{2}\right)$ & -0.2593 & 0.1671 & -0.3052 & 0.1970 \\
& $\left(y_{2}, x_{2}\right)$ & 0.2700 & 0.2112 & 0.1454 & 0.2308 \\
& $\left(y_{2}, y_{1}\right)$ & 0.5752 & 0.1360 & 0.0919 & 0.2473 \\
\hline $\boldsymbol{\Sigma}_{2}$ & $\left(y_{1}, y_{1}\right)$ & -0.1070 & 0.1547 & -0.5843 & 0.3830 \\
& $\left(y_{2}, y_{2}\right)$ & -0.4106 & 0.2347 & -0.3392 & 0.3889 \\
& $\left(y_{2}, y_{1}\right)$ & -0.2375 & 0.1319 & -0.3370 & 0.2411 \\
\hline & & & & &
\end{tabular}

